\title{
Efecto de fluoxetina y dieta baja en carbohidratos en marcadores de riesgo cardiometabólico en sujetos con riesgo de diabetes mellitus
}

Effect of fluoxetine and a low-carbohydrate diet on cardiometabolic risk markers in subjects at risk of diabetes mellitus

\author{
Janet Del R. Gordillo-Cortaza \\ Sócrates D. Pozo-Verdesoto \\ Gisella K. Sanclemente-Lainez \\ Giomar R. Viteri-Gomez \\ Yuliana Y. Gomez-Rutti \\ Fátima V. Feraud-Ibarra \\ Nancy A. Vasquez-Rodriguez \\ Walter A. Gonzalez-García \\ Shirley Poveda-Navarrete
}




\title{
Efecto de fluoxetina y dieta baja en carbohidratos en marcadores de riesgo cardiometabólico en sujetos con riesgo de diabetes mellitus
}

\author{
Effect of fluoxetine and a low-carbohydrate diet on cardiometabolic risk \\ markers in subjects at risk of diabetes mellitus
}

Janet Del R. Gordillo-Cortaza ${ }^{1}$, Socrates D. Pozo-Verdesoto ${ }^{2}$, Gisella K. SanclementeLainez $^{3}$ Giomar R. Viteri-Gomez ${ }^{4}$ Yuliana Y. Gomez-Rutti ${ }^{5}$, Fatima V. Feraud-Ibarra ${ }^{6}$, Nancy A. Vasquez- Rodriguez ${ }^{7}$, Walter A. Gonzalez-Garcia ${ }^{8}$, Shirley Poveda-Navarrete ${ }^{9}$

\section{RESUMEN}

Antecedentes: Las dietas bajas en carbohidratos son una buena estrategia para disminuir el peso, regular los niveles de insulina y los parámetros antropométricos en pacientes con obesidad con perfil cardiovascular adverso. Objetivo: Determinar el efecto de la fluoxetina con una dieta baja en carbohidratos en sujetos con riesgo de diabetes mellitus. Material y Métodos: Se realizó un estudio observacional y retrospectivo, se revisó 162 historias clínicas del Servicio de Nutrición del Hospital Universitario Guayaquil; se seleccionaron a 81 pacientes que siguieron una dieta baja en carbohidratos < de $130 \mathrm{~g} /$ día y 81 pacientes con la misma dieta más una dosis diaria de $40 \mathrm{mg}$ de fluoxetina. Se evaluaron parámetros antropométricos y bioquímicos, al inicio y 6 meses después de la intervención. Para el análisis estadístico, se aplicó la prueba de Wilcoxon y la prueba de Wald-Wolfowitz. Resultados: Los pacientes tratados con dieta baja en carbohidratos y fluoxetina, tuvieron una disminución significativa del peso $(\Delta=5,85 \mathrm{~kg}$; $\mathrm{p}<0,001)$, a diferencia de los pacientes tratados con una dieta baja en carbohidratos. Similares resultados se obtuvieron al comparar el IMC $(\Delta=2,54 \mathrm{~kg} / \mathrm{m} 2 ; \mathrm{p}<0,001)$, la insulina $(\Delta=2,62 ; \mathrm{p}<0,001)$, el HOMA-IR $(\Delta=0,81$; $\mathrm{p}<0,001)$ y los valores metabólicos de colesterol y glucosa, $(\mathrm{p}<0,001)$ Conclusión Los pacientes tratados con fluoxetina y dieta baja en carbohidratos disminuyeron los niveles de los marcadores de riesgo cardiometabólico en sujetos de riesgo con diabetes mellitus.

Palabras clave: Fluoxetina; Dieta; Diabetes mellitus. (Fuente:DeCS-BIREME)

\footnotetext{
${ }^{1}$ Especialista en nutrición, PhD en ciencias de la salud, Docente de la Facultad de Medicina, Universidad de Guayaquil, Ecuador. Correo electrónico: janetgordillo28@gmail.com.

${ }^{2}$ Magister en Salud Publica y en Nutrición, Docente de la Facultad de Medicina, Universidad de Guayaquil, Ecuador. Correo electrónico: socratespozo@hotmail.com

${ }^{3}$ Especialista en nutrición, Servicio de Nutrición y Dietética, Universidad de Guayaquil, Ecuador. Correo electrónico: drasanclemente@hotmail.com

${ }^{4}$ Magister en atención Primaria, Docente de Universidad Privada, Hospital Universitario de Guayaquil, Ecuador. Correo electrónico: giomar.viteri@hug.gob.ec

${ }^{5}$ Magister en Nutrición Publica, Docente de Universidad Privada, Universidad de Guayaquil, Ecuador. Correo electrónico: yuliana.gomez@upn.pe

${ }^{6}$ Especialista en Medicina Interna, Docente de la Facultad de Medicina, Hospital Universitario de Guayaquil, Universidad de Guayaquil, Ecuador. Correo electrónico: draferaud@gmail.com

${ }^{7}$ Especialista en Medicina Interna, Docente de la Facultad de Medicina, Universidad de Guayaquil, Ecuador. Correo electrónico: nancyvr0@ hotmail.com

${ }^{8}$ Doctor en Nutrición, Docente de la Facultad de Ciencias de la Salud, Universidad Técnica de Babahoyo, Ecuador. Correo electrónico: walteradal@yahoo.es.

${ }^{9}$ Estudiante de la Carrera de Dietética y Nutrición Constitucional, Estudiante de la Escuela de Nutrición, Universidad de Guayaquil, Ecuador. Correo electrónico: shirleypoveda92@gmail.com
} 


\begin{abstract}
Background: Low carbohydrate diets are a good strategy to reduce weight, regulate insulin levels and anthropometric parameters in obese patients with an adverse cardiovascular profile. Objective: To determine the effect of fluoxetine, with a low carbohydrate diet in subjects at risk of diabetes mellitus. Materials and Methods: An observational and retrospective study was carried out, 162 medical records of the Nutrition Service at the University Hospital Guayaquil, were reviewed. 81 patients who followed a low carbohydrate diet $<130 \mathrm{~g} /$ day and 81 patients with the same diet plus a daily dose of $40 \mathrm{mg}$ of fluoxetine were selected. Anthropometric and biochemical parameters were evaluated at the beginning and 6 months after the intervention. For statistical analysis, the Wilcoxon test and the WaldWolfowitz test were applied. Results: The patients treated with a low-carbohydrate diet and fluoxetine had a significant decrease in weight $(\Delta=5.85 \mathrm{~kg}$; $\mathrm{p}<0.001)$, unlike the patients treated with a low-carbohydrate diet. Similar results were obtained when comparing BMI $(\Delta=2.54 \mathrm{~kg} / \mathrm{m} 2 ; \mathrm{p}<0.001)$, insulin $(\Delta=2.62 ; \mathrm{p}<0.001)$, HOMA-IR $(\Delta=0.81 ; \mathrm{p}<0.001)$ and the metabolic values of cholesterol and glucose, $(\mathrm{p}<0.001)$. Conclusion Patients treated with fluoxetine and low carbohydrate diet improve cardio metabolic risk markers, in subjects at risk with diabetes mellitus.
\end{abstract}

Key words: Fluoxetine; Diet; Mellitus Diabetes (Source:DeCS-BIREME).

\title{
INTRODUCCIÓN
}

La Organización Mundial de la Salud (OMS) refiere que más de 40 millones de personas mueren anualmente por Enfermedades Crónicas no Transmisibles (ECNT) las principales causas de muerte son las enfermedades cardiovasculares (17,7 millones cada año), seguidas del cáncer (8,8 millones), enfermedades respiratorias (3,9 millones) y diabetes (1,6 millones) (1) así mismo alrededor del 20 al 30\% de la población en general de muchos países podría estar sufriendo de síndrome metabólico ${ }^{(2,3)}$.

Según el Instituto de Estadísticas y Censos (INEC) 2018, cinco de las diez principales causas de fallecimientos en Ecuador se relacionan con estos malos hábitos alimenticios, las enfermedades no transmisibles representaron el 53\% del total de las muertes, de estas, el $48,6 \%$ correspondió a enfermedades cardiovasculares, el $30 \%$ a cáncer, el 12,4\% a diabetes y el $8,7 \%$ a enfermedades respiratorias crónicas ${ }^{(4)}$ que puede incrementar de dos a cuatro veces el riesgo de insuficiencia cardíaca en comparación con individuos no diabéticos ${ }^{(5,6)}$. El estrés genera un incremento de la producción de cortisol, un glucocorticoide que podría influir en la conducta e ingesta de alimentos con alto índice glicémico ${ }^{(7)}$. El eje del hipotálamo-hipófisis-adrenal, regula las concentraciones en el plasma, considerado un disruptor de la regulación del apetito ${ }^{(8)}$, dando lugar a una serie de mecanismos que favorecen la compulsión alimentaria, generando un aporte calórico sobredimensionado a los requerimientos energéticos.

La fluoxetina es un antidepresivo, inhibidor selectivo de la recaptación de serotonina (ISRS), su uso en el tratamiento de algunos trastornos alimenticios ha sido justificado en los desórdenes alimentarios (BED), por su implicación en la regulación de las conductas alimentarias o compulsiones a comer ${ }^{(9,10)}$. La prescripción de regímenes bajos en carbohidratos es utilizada en pacientes con obesidad o riesgo de diabetes mellitus, una dieta baja en carbohidratos, que represente $130 \mathrm{~g}$ /día, podría actuar sinérgicamente con el uso de la fluoxetina para tratar los trastornos obsesivo compulsivo y evitar recaídas en la pérdida de peso ${ }^{(11)}$.

Las variables ambientales como dieta y estilo de vida influyen directamente sobre la composición corporal, convirtiéndose en un componente más fuerte que el genético. En 
efecto, las dietas saludables ocasionan cambios favorables sobre los marcadores de riesgo cardiometábolico ${ }^{(12)}$. Varias entidades como la OMS, la American Association of Clinical Endocrinologists (AACE), International Diabetes Federation (IDF) y el National Cholesterol Education Program Adult Treatment Panel III (ATP III) han establecido criterios que definen la situación de riesgo de padecer Síndrome Metabólico (Tabla 1), la mayoría pueden ser modificables a través de un estilo de vida saludable ${ }^{(13)}$.

Un estudio sobre asociaciones positivas entre el IMC y el índice glicémico sugiere que el consumo de carbohidratos desempeña un papel importante en la determinación del peso corporal $^{(14)}$. La variabilidad glicémica no solo está afectada por la calidad y cantidad de carbohidratos ${ }^{(15)}$, también por la ingesta de proteínas y fibras, con implicaciones importantes de riesgo de diabetes, inflamación y otros ${ }^{(16)}$. Además, mejoraría la proporción calórica relativa proveniente de las proteínas y grasas que lleva a una mejor saciedad ${ }^{(17,18)}$. Las dietas con índice glucémico bajo puede causar disminuciones significativas en las medidas antropométricas, presión arterial, glucosa y perfiles de lípidos séricos (Triglicéridos, colesterol total y niveles bajos de colesterol de lipoproteínas de densidad (LDL-C) y colesterol unido a lipoproteínas de alta densidad; (HDL-C) ${ }^{(19,20,21)}$. En el manejo de dietas modificadas en razón a la ingesta de carbohidratos, Feinman et al sugieren definiciones de dietas, entre ellas la de consumo de carbohidratos menor a 130 gramos por día ${ }^{(22)}$.

\section{MATERIALES Y MÉTODOS}

Se realizó un estudio analítico, comparativo, longitudinal y retrospectivo, mediante la revisión de 162 historias clínicas, de pacientes atendidos en la Consulta Externa del Servicio de Nutrición del Hospital Universitario de la ciudad de Guayaquil, entre junio a diciembre del 2019, con diagnóstico de obesidad y que presentaban al menos tres componentes del síndrome metabólico un IMC desde $\geq 29$, HOMA IR y perímetro abdominal. Se empleó un muestreo no probabilístico, por conveniencia, se excluyeron a pacientes cuya historia clínica incluyera diagnósticos de diabetes mellitus, trastornos de la conducta alimentaria y aquellas que no constaban con todos los parámetros evaluados para el presente estudio. Se conformaron dos grupos de estudio (dieta + fluoxetina y dieta). Se evaluaron parámetros antropométricos: peso (kg), el índice de masa corporal (IMC) (23), el perímetro abdominal (cm) (24); los biomarcadores serológicos; colesterol total $(\mathrm{mg} / \mathrm{dl})$, triglicéridos $(\mathrm{mg} / \mathrm{dl})$ y glicemia en ayunas $(\mathrm{mg} / \mathrm{dl})$, se midieron con los Estuches Reactivos colorímetricos de Trinder; La insulina basal, se realizó por quimioluminiscencia con reactivo cuantificado; el Homa-IR modelo homeostático (25).

\section{Análisis Estadístico}

Los datos fueron recogidos en una hoja de cálculo de Microsoft Excel, y exportados luego al Programa SPSS versión 25.

El análisis de datos se realizó bajo un intervalo de confianza de $95 \%$ y un valor $\mathrm{p}<0,05$ se consideró estadísticamente significativo. Se calculó el promedio, desviación estándar de las variables investigadas, y se utilizó la pruebas de normalidad de Kolmogorov Smirnov modificado por Lilliford, y posterior a ello, se utilizó la prueba de Signos de Wilcoxon, para comparar el antes y después en los grupos de dieta baja en carbohidratos y Fluoxetina, y solo dieta baja en carbohidratos, posteriormente se calculó la diferencia entre los valores antes y después de la intervención, de las variables antropométricas y bioquímicas, y se determinó su diferencial por la prueba de Kruskall Wallis (basado en las pruebas de 
normalidad de las variables numéricas con la prueba de Kolmorov Smirnov Lilliford) para determinar la diferencia de cada parámetro según el tratamiento recibido.

\section{RESULTADOS}

Las historias clínicas de los pacientes tenían edades de $43 \pm 13$ años, el $92.6 \%$ de los participantes eran mujeres y el resto estuvo conformado por varones (Tabla 2). El estudio se dividió en dos grupos, 81 pacientes estuvieron en tratamiento durante 6 meses con una dieta considerada baja en carbohidratos $3 \mathrm{~g} / \mathrm{kg}$ peso corporal/día y 81 pacientes que además de la dieta baja en carbohidratos, recibieron una dosis diaria de $40 \mathrm{mg}$ de fluoxetina.

Los pacientes que tuvieron un tratamiento basado en una dieta baja en calorías y fluoxetina (tabla 3), se observa que el peso medio inicial se estableció en $93.52 \pm 18,18 \mathrm{~kg}$. Luego del tratamiento se identificó una disminución de peso, obteniéndose $87.67 \pm 18,16 \mathrm{~kg}$. Se aplicó la prueba de Wilcoxon y se encontró una diferencia estadísticamente significativa ( $\mathrm{p}<0.001)$. En cuanto a los valores medios de los parámetros medidos como el IMC (inicial: 38,98 \pm 6,39; final: $36,44 \pm 6,09 ; \mathrm{p}<0.001$ ), el perímetro abdominal (inicial: 116,32 $\pm 14,33 \mathrm{~cm}$; final: $111,16 \pm 14,22 \mathrm{~cm} ; \mathrm{p}<0.001$ ), en el diagrama de cajas muestra un cambio significativo en las pacientes con dieta + fluoxetina (Gráfico 1), la concentración de insulina $(21,23 \pm 6,44$

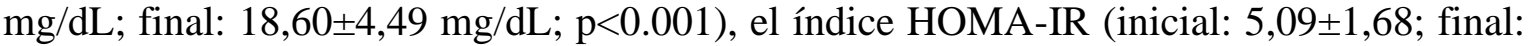
4,27 $\pm 1,08 ; \mathrm{p}<0.001$ ), los triglicéridos (inicial: $172,33 \pm 88,05 \mathrm{mg} / \mathrm{dL}$; final: $137,22 \pm 34,85$ $\mathrm{mg} / \mathrm{dL}$; p<0.001) y la glucosa (inicial: $94,87 \pm 7,44 \mathrm{mg} / \mathrm{dL}$; final: $90,55 \pm 8,34 \mathrm{mg} / \mathrm{dL}$; $\mathrm{p}<0.001)$ disminuyeron significativamente, con excepción de los valores de colesterol el cual no tuvo una disminución significativa $(\mathrm{p}=0,07)$ ante la intervención dietética $\mathrm{y}$ medicamentosa (Gráfico 1).

En el caso de los pacientes que fueron tratados solo con dieta baja en carbohidratos (Tabla 4) se observó que el peso también disminuyó durante los seis meses de evaluación. El diferencial fue $1,21 \pm 2,26 \mathrm{~kg}$, siendo significativa esta variación ( $\mathrm{p}=0,03)$; en el caso del índice de masa corporal, el diferencial fue $0,83 \pm 1,33 \mathrm{~kg} / \mathrm{m} 2$ en promedio siendo significativa la prueba de Wilcoxon $(\mathrm{p}<0.001)$. En el caso del perímetro abdominal (diferencial: $5,00 \pm 4,49 ; \mathrm{p}=1,00$ ), la insulina (diferencial: $-17,32 \pm 13,070,03 ; \mathrm{p}<0.001$ ), el índice HOMAIR (diferencial: $0,91 \pm 3,23 ; \mathrm{p}<0.001$ ) y la glucosa (diferencial: $4,74 \pm 7,62 ; \mathrm{p}=1,00$ ), estos se redujeron significativamente, aunque en menor cuantía, comparando con el grupo de dieta más fluoxetina, con excepción de los valores de triglicéridos y colesterol respectivamente, los cuales fueron significativos ante la prueba de Wilcoxon.

Posteriormente se realizó un análisis paramétrico de las variables numéricas antes mencionadas. Se concluyó que, tanto las variables y sus diferencias antes y después de la intervención, sobre el peso, IMC, perímetro abdominal, insulina, índice HOMA-IR, triglicéridos y colesterol no presentaban una distribución normal ante la prueba de Kolmogorov-Smirnov, por lo cual se optó por la prueba de Wald Wolfowitz, para determinar la diferencia que existe entre los valores iniciales y finales de cada variable antropométrica y bioquímica, en relación a los dos grupos (dieta con fluoxetina y de la dieta únicamente). Se observó que la diferencia del peso de los pacientes sometidos a dieta y fluoxetina presenta un promedio de $5,85 \pm 4,50 \mathrm{~kg}$ y para el grupo dietético únicamente esta diferencia fue menor con 1,21 $\pm 2,26 \mathrm{~kg}$ por tanto, ante la prueba de estadísticas estas son significativas $(\mathrm{p}=0,03$ ) para la diferencia del peso.(Tabla 5) en el caso del índice de masa corporal los diferenciales son de $2,54 \pm 2,02 \mathrm{~kg} / \mathrm{m} 2$ para el grupo de dieta y fluoxetina y presenta un valor de $0,83 \pm 1,33$ $\mathrm{kg} / \mathrm{m} 2$ para el grupo de las dietas, siendo significativa $(\mathrm{p}<0,01)$ la diferencia a favor de los grupo de la dieta y fluoxetina. 
En el caso de la diferencia de la concentración de insulina, obtenemos valores de -15,21 para el grupo de dieta y fluoxetina y el diferencial con los grupos de la dieta en - 17,32, lo cual muestra que esta diferencia es estadísticamente significativa a favor de mejorar los niveles de insulina con el grupo de dieta y fluoxetina. En el caso del índice HOMA-IR se obtuvo valores de 0,82 con el grupo de dieta y fluoxetina, a diferencia de 0,91 , que se obtiene a favor de la dieta, por lo que también presentan una diferencia significativa entre las pruebas estadísticas a favor del grupo de dieta y fluoxetina.

\section{Discusión}

Varios estudios han asociado las dietas bajas en carbohidratos a una mejor respuesta de los parámetros bioquímicos, triglicéridos, colesterol, y pérdida de peso ${ }^{(20,21,26)}$ en sujetos con obesidad o con diabetes mellitus tipo 2. También refieren que mejoran los niveles de glucosa en sangre ${ }^{(27)}$, esto coincide con los hallazgos obtenidos en el presente estudio al administrar una dieta baja en carbohidratos, donde esta disminución resultó ser significativa ${ }^{(28) .}$

Por otro lado, la fluoxetina, es un fármaco inhibidor selectivo de la recaptación de la serotonina, que puede promover una reducción en la ingesta de alimentos, con lo que se conseguiría disminuir el peso corporal ${ }^{(29)}$, existen estudios que no evidencian pérdida de peso significativa en aquellos pacientes tratados con este fármaco ${ }^{(30)}$. Sin embargo, un estudio de metaanálisis incluyó estudios que tuvieron como intervención a la fluoxetina con dosis de $60 \mathrm{mg}$. en pacientes adultos, promoviendo pérdidas de peso entre $0.9 \mathrm{~kg}$. a $9.1 \mathrm{~kg}$. durante los primeros meses, sin embargo, la reducción del peso dejó de ser significativa a los 12 meses de intervención, comparándola con placebo. Asimismo, se identificaron eventos adversos como nerviosismo, náuseas y vómitos, fatiga, insomnio, sudoración y temblores ${ }^{(31)}$, estos resultados concuerdan con los resultados del presente estudio.

Por otra parte, un estudio realizado por Guisado-Macías, muestra que la pérdida media de peso a los 3 meses de administración de fluoxetina fue de $1.1 \mathrm{~kg}$ (rango: 0-24) y de 7,6 kg a los 6 meses (rango: 0-19) ${ }^{(32)}$. Es necesario mencionar que el efecto este fármaco es mejor si su administración no se da en forma de monoterapia ${ }^{(32)}$, el estudio no incluye el análisis estadístico a las variables confusoras, puede existir un sesgo de intención a tratar, porque son personas con intención a perder peso y de acceder a una cirugía, es probable que el efecto en la pérdida de peso no sea exclusivamente atribuible a los fármacos, sino quizá a la actividad física o un régimen dietético.

Por otro lado, las dietas bajas en carbohidratos podrían reducir el uso de medicamentos para el control de la glucemia en pacientes con diabetes tipo $2^{(33)}$. Darga L, refiere que cuando administró una dieta baja en carbohidratos, obtuvo una disminución significativa de peso máximo de $12.4 \mathrm{~kg}$ en la semana 29 de tratamiento, en sujetos tratados con fluoxetina y dieta ${ }^{(34)}$, que fue significativamente mayor que la obtenida con dieta y placebo. No obstante, al final del estudio, en la semana 45, la pérdida de peso llegó a ser de 8,2 $\mathrm{kg}$ y continúo siendo mayor en el grupo que además recibía fluoxetina, pero esta diferencia ya no resultó ser

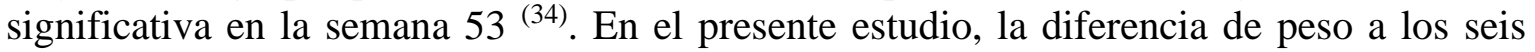
meses de administrar una dieta baja en carbohidratos y fluoxetina, resultó ser significativamente diferente a la disminución de peso obtenida solo con dieta, se logró una disminución máxima de $5,7 \mathrm{~kg}$.

Al igual que en algunos estudios realizados con dieta baja en hidratos de carbohidratos ${ }^{(35)}$, se observó disminución significativa para los parámetros bioquímicos investigados como triglicéridos, colesterol total y glucosa, también en los parámetros metabólicos: insulina e índice HOMA-IR. Esta disminución se observó en los dos tipos de tratamiento, pero no representó diferencia significativa cuando se compararon ambos. 


\section{CONCLUSIÓN}

Este hallazgo sugiere que el uso de fluoxetina juntamente con una dieta baja en carbohidrato es efectiva para perder y mantenerlo, es útil también para mejorar los niveles de factores de riesgo metabólico como la insulina plasmática y el HOMA-IR, mientras que estos hallazgos preliminares son prometedores, todavía requieren una confirmación por un ensayo controlado aleatorizado a mayor escala.

\section{Aspectos éticos}

El protocolo del estudio fue aprobado por Comité Interno de Ética en Investigación del Hospital Universitario de Guayaquil - Ecuador, antes de desarrollar el estudio. Para asegurar la confidencialidad de los datos, se asignaron identificadores codificados aleatoriamente y se obtuvo los datos de los pacientes.

\section{Conflictos de interés}

Los autores declaran no tener conflicto de intereses

\section{Financiamiento}

Autofinanciado

\section{REFERENCIAS}

World Health Organization. UN News. Urgent health challenges for the next decade according to WHO. Ginebra. OMS ;2010. Disponible en: https://teresaperez.net/2020/01/21/retos-de-salud-urgentes-para-la-proximadecada-segun-la-oms/.

Van Vliet-Ostaptchouk JV, Nuotio M-L, Slagter SN, Doiron D, Fischer K, Foco L, et al. The prevalence of metabolic syndrome and metabolically healthy obesity in Europe: a collaborative analysis of ten large cohort studies. BMC Endocr Disord. 2014;14(1):9. https://doi.org/10.1186/1472-6823-14-9.

Xi B, He D, Hu Y, Zhou D. Prevalence of metabolic syndrome and its influencing factors among the Chinese adults: the China health and nutrition survey in 2009. Prev Med (Baltim). 2013;57(6):867-71. https://doi. org/10.1016/j.ypmed.2013.09.023

Encuesta STEPS Ecuador 2018 MSP, INEC, OPS/OMS. Vigilancia de enfermedades no transmisibles y factores de riesgo. 2018. https://www.salud.gob.ec/wpcontent/uploads/2020/10/INFORME-STEPS.pdf

Aneja A, Tang WH, Bansilal S, Garcia MJ, Farkouh ME. Diabetic cardiomyopathy: insights into pathogenesis, diagnostic challenges, and therapeutic options. Am J Med. 2008 Sep;121(9):748-57. Doi: 10.1016/j.amjmed.2008.03.046.

Steele, J.M., Urbina, E.M., Mazur, W.M. et al. Left atrial strain and diastolic function abnormalities in obese and type 2 diabetic adolescents and young adults. Cardiovasc Diabetol 19, 163 (2020). https://doi.org/10.1186/s12933-020-01139-9

Van der Valk ES, Savas M, van Rossum EFC. Stress and Obesity: Are There More Susceptible Individuals? Curr Obes Rep. 2018 Jun;7(2):193-203. Doi: 10.1007/s13679-018-0306-y.

Cortés Romero Celso Enrique, Escobar Noriega Alejandra, Cebada Ruiz Jorge, Soto Rodríguez Guadalupe, Bilbao Reboredo Tania, Vélez Pliego Marcela. Estrés y cortisol: implicaciones en la ingesta de alimento. Rev Cubana Invest Bioméd. 2018; 37(3): 1-15. Disponible en: http://scielo.sld.cu/scielo.php?script=sci_arttext\&pid=S086403002018000300013\&lng=es. 
McElroy SL, Guerdjikova AI, Mori N, O'Melia AM. Pharmacological management of binge eating disorder: current and emerging treatment options. Ther Clin Risk Manag. 2012;8:219-41. Doi: 10.2147/TCRM.S25574.

Devlin MJ, Goldfein JA, Petkova E, Jiang H, Raizman PS, Wolk S, Mayer L, Carino J, Bellace D, Kamenetz C, Dobrow I, Walsh BT. Cognitive behavioral therapy and fluoxetine as adjuncts to group behavioral therapy for binge eating disorder. Obes Res. 2005 Jun;13(6):1077-88. Doi: 10.1038/oby.2005.126. PMID: 15976151.

Gorla K y Mathews M.Pharmacological Treatment of Eating Disorders. Psychiatry (Edgmont). 2005 Jun; 2(6): 43-48. Disponible en: https://www.ncbi.nlm.nih.gov/pmc/articles/PMC3000192/

Díaz Anibal. Factores de riesgo cardiovascular y disfunción endotelial en adultos que viven a gran altura. Acta méd. Peru . 2016; 33(4): 289-295. Disponible en: http://www.scielo.org.pe/scielo.php?script=sci_arttext\&pid=S172859172016000400005\&lng=es.

Beigi I, Moghisi F, Inzucchi S, Tiktin M. Standards Medical care in Diabetes. American Diabetes Association. 2012 March; 35(3). https://doi.org/10.2337/dc12-s011

Vega-López S, Venn BJ, Slavin JL. Relevance of the Glycemic Index and Glycemic Load for Body Weight, Diabetes, and Cardiovascular Disease. Nutrients. 2018 Sep 22;10(10):1361. Doi: 10.3390/nu10101361.

Tay J, Thompson CH, Brinkworth GD. Glycemic Variability: Assessing Glycemia Differently and the Implications for Dietary Management of Diabetes. Annu Rev Nutr. 2015;35:389-424. Doi: 10.1146/annurev-nutr-121214-104422.

Weigle DS, Breen PA, Matthys CC, Callahan HS, Meeuws KE, Burden VR, Purnell JQ. A high-protein diet induces sustained reductions in appetite, ad libitum caloric intake, and body weight despite compensatory changes in diurnal plasma leptin and ghrelin concentrations. Am J Clin Nutr. 2005 Jul;82(1):41-8. Doi: 10.1093/ajcn.82.1.41.

Fechner E, Bilet L, Peters HPF, Hiemstra H, Jacobs DM, Op 't Eyndt C, Kornips E, Mensink RP, Schrauwen P. Effects of a whole diet approach on metabolic flexibility, insulin sensitivity and postprandial glucose responses in overweight and obese adults - A randomized controlled trial. Clin Nutr. 2020 Sep;39(9):2734-2742. Doi: 10.1016/j.clnu.2019.12.010.

Trumbo P, Schlicker S, Yates AA, Poos M; Food and Nutrition Board of the Institute of Medicine, The National Academies. Dietary reference intakes for energy, carbohydrate, fiber, fat, fatty acids, cholesterol, protein and amino acids. J Am Diet Assoc. 2002 Nov;102(11):1621-30. Doi: 10.1016/s0002-8223(02)90346-9.

Rajabi S, Mazloom Z, Zamani A, Tabatabaee HR. Effect of low glycemic index diet versus metformin on metabolic syndrome. Int J Endocrinol Metab. 2015;13(4):e23091. https://doi.org/10.5812/ijem.23091

Goldenberg JZ, Day A, Brinkworth GD, Sato J, Yamada S, Jönsson T, Beardsley J, Johnson JA, Thabane L, Johnston BC. Efficacy and safety of low and very low carbohydrate diets for type 2 diabetes remission: systematic review and meta-analysis of published and unpublished randomized trial data. BMJ. 2021 Jan 13;372:m4743. doi: 10.1136/bmj.m4743.

Barber TM, Hanson P, Kabisch S, Pfeiffer AFH, Weickert MO. The Low-Carbohydrate Diet: ShortTerm Metabolic Efficacy Versus Longer-Term Limitations. Nutrients. 2021 Apr 3;13(4):1187. doi: 10.3390/nu13041187.

Feinman R, Pogozelski W, Astrup A, Bernstein R, Fine EECWE. Dietary carbohydrate restriction as the first approach in diabetes management: critical review and evidence base. Nutrition. 2015 January; 31(1). Doi: 10.1016/j.nut.2014.06.011. 
Organization WH. Reports of a WHO Consultation. [Online].; 2003 [cited 2020 agosto sabado. Available from: www.who.int/nutrition/publications/obesity/WHO_TRS_894/en/

Shetty P, Kumanyika S. Waist Circumference and Waist-Hip Ratio. Report of a WHO Expert Consultation. $2010 \quad$ January; https://www.who.int/publications/i/item/9789241501491

Hernández Yero José Arturo, Tuero Iglesias Ángela, Vargas González David. Utilidad del índice HOMA-IR con una sola determinación de insulinemia para diagnosticar resistencia insulínica. Rev Cubana Endocrinol . 2011 ; 22(2): 69-77. Disponible en: http://scielo.sld.cu/scielo.php?script=sci_arttext\&pid=S156129532011000200002\&lng=es.

Feinman RD, Pogozelski WK, Astrup A, et al. La restricción de carbohidratos en la dieta como primer enfoque en el manejo de la diabetes: revisión crítica y base de evidencia . Nutrición 2015; 31 : 1-13. 10.1016 / j.nut.2014.06.011

Brinkworth G, Noakes M, Buckley J, Keogh J. Long-term effects of a very-lowcarbohydrate weight loss diet compared with an isocaloric low-fat diet after 12 months. Am J Clin Nutr. 2009 July; 90(1). Doi: 10.3945/ajcn.2008.27326.

Napoleão, A.; Fernandes, L.; Miranda, C.; Marum, A.P. Effects of Calorie Restriction on Health Span and Insulin Resistance: Classic Calorie Restriction Diet vs. KetosisInducing Diet. Nutrients 2021, 13, 1302. https://doi.org/10.3390/ nu13041302

Serralde-Zúñiga AE, Gonzalez Garay AG, Rodríguez-Carmona Y, Melendez G. Fluoxetina para adultos con sobrepeso u obesidad. Cochrane Database Syst Rev.2019 15 de octubre; 10 (10): CD011688. doi: 10.1002 / 14651858.CD011688.

Suplicy H, Boguszewski CL, Santos CM, Desterro de Figueiredo M, Cunha DR, Radominski R. A comparative study of five centrally acting drugs on the pharmacological treatment of obesity. Int J Obes (Lond). 2014;38(8):1097-103. Doi:10.1038/ijo.2013.225

Li Z, Maglione M, Tu W, Mojica W, Arterburn D, Shugarman LR, Hilton L, Suttorp M, Solomon V, Shekelle PG, Morton SC. Meta-analysis: pharmacologic treatment of obesity. Ann Intern Med. 2005 Apr 5;142(7):532-46. Doi: 10.7326/0003-4819-1427-200504050-00012.

Guisado-Macías J, Méndez F, Baltasar I, Zamora F. Fluoxetina, topiramato y combinación de ambos en el control de la conducta alimentaria antes de la cirugía bariatrica. Actas Esp Psiquiatr. 2016; 44(3). Disponible en https://actaspsiquiatria.es/repositorio/18/101/ESP/18-101-ESP-93-6-683908.pdf

Hall KD, Chung ST. Low-carbohydrate diets for the treatment of obesity and type 2 diabetes. Curr Opin Clin Nutr Metab Care. 2018 Jul;21(4):308-312. Doi: 10.1097/MCO.0000000000000470.

Darga LL, Carroll-Michals L, Botsford SJ, Lucas CP. Fluoxetine's effect on weight loss in obese subjects. Am J Clin Nutr. 1991 Aug;54(2):321-5. Doi: 10.1093/ajcn/54.2.321.

Brouns F. Overweight and diabetes prevention: is a low-carbohydrate-high-fat diet recommendable? Eur J Nutr. 2018 Jun;57(4):1301-1312. Doi: 10.1007/s00394-0181636-y. 


\section{Anexos}

Tabla 1.

Componentes del síndrome metabólico considerando su definición, según la National Cholesterol Education Program Adult Treatment Panel III (ATP III). Organización Mundial de la Salud (OMS). American Association of Clinical Endocrinologists (AACE), International Diabetes Federation $\left(I D F^{(13)}\right.$

\begin{tabular}{|c|c|c|c|c|}
\hline Componentes & ATP III & OMS & AACE & IDF \\
\hline Triglicérido mayor o igual a $150 \mathrm{mg} / \mathrm{dl}$ & $\mathrm{X}$ & $\mathrm{X}$ & $\mathrm{X}$ & $\mathrm{X}$ \\
\hline $\begin{array}{l}\text { HDL menor de } 40 \mathrm{mg} / \mathrm{dl} \text { en varones y } 50 \\
\mathrm{mg} / \mathrm{dl} \text { en mujeres }\end{array}$ & $\mathrm{X}$ & $X$ & $X$ & $\mathrm{X}$ \\
\hline Presión arterial mayor de 130/85 mmHg & $\mathrm{X}$ & $\mathrm{X}$ & $\mathrm{X}$ & $\mathrm{X}$ \\
\hline Insulino resistencia (IR) & & $\mathrm{X}$ & & \\
\hline Glucosa en ayunas mayor de $100 \mathrm{mg} / \mathrm{dl}$ & $\mathrm{X}$ & & $\mathrm{X}$ & $X$ \\
\hline Glucosa $2 \mathrm{~h}: 140 \mathrm{mg} / \mathrm{dl}$ & & & $\mathrm{X}$ & \\
\hline Obesidad abdominal & $\mathrm{X}$ & & & $X$ \\
\hline Índice de masa corporal elevado & & $\mathrm{X}$ & $\mathrm{X}$ & \\
\hline Microalbuminuria & & $\mathrm{X}$ & & \\
\hline Factores de riesgo y diagnóstico & $\begin{array}{l}3 \text { más } \\
\text { IR }\end{array}$ & $\begin{array}{l}\text { Más } \\
\text { de } 2\end{array}$ & $\begin{array}{l}\text { Criterio } \\
\text { clínico }\end{array}$ & $\begin{array}{l}\text { Obesidad } \\
\text { abdominal }\end{array}$ \\
\hline
\end{tabular}

Tabla 2.

Datos sobre edad y sexo.

\begin{tabular}{lllll}
\hline \multirow{2}{*}{ Grupos } & \multirow{2}{*}{ Edad $(\underline{x} \pm$ D.E. $)$} & \multicolumn{2}{l}{ Sexo $(\%(\mathrm{n}))$} \\
\cline { 3 - 5 } & & $43,41 \pm 12,79$ & Mujeres & Varones \\
\hline Total & (Dieta+ & $43,47 \pm 12,31$ & $46.3(75)$ & $7,4(12)$ \\
frupo 1 & & $4,7(6)$ \\
Grupotina) & & $43,35 \pm 13,33$ & $46.3(75)$ & $3,7(6)$ \\
\hline
\end{tabular}


Tabla 3.

Parámetros antropométricos y bioquímicos antes del inicio del tratamiento y después de seis meses de tratamiento con Fluoxetina y dieta.

\begin{tabular}{|c|c|c|c|c|c|}
\hline \multirow{2}{*}{ Parámetros } & \multirow{2}{*}{$\begin{array}{l}\text { Antes } \\
\text { Promedio }\end{array}$} & \multirow[b]{2}{*}{ D.E. } & \multirow{2}{*}{$\frac{\text { Después }}{\text { Promedio }}$} & \multirow[b]{2}{*}{ D.E. } & \multirow[b]{2}{*}{$\mathbf{p}^{*}$} \\
\hline & & & & & \\
\hline Peso (kg) & 93,52 & 18,18 & 87,67 & 18,16 & $<0.001$ \\
\hline $\begin{array}{l}\text { Índice de masa } \\
\text { corporal }\end{array}$ & 38,98 & 6,39 & 36,44 & 6,09 & $<0.001$ \\
\hline $\begin{array}{l}\text { Perímetro abdominal } \\
(\mathrm{cm})\end{array}$ & 116,32 & 14,33 & 111,16 & 14,22 & $<0.001$ \\
\hline Insulina (mg/dL) & 21,23 & 6,44 & 18,60 & 4,49 & $<0.001$ \\
\hline HOMA-IR & 5,09 & 1,68 & 4,27 & 1,08 & $<0.001$ \\
\hline Triglicéridos (mg/dL) & 172,33 & 88,05 & 137,22 & 34,85 & $<0.001$ \\
\hline Colesterol (mg/dL) & 194,92 & 47,63 & 181,32 & 28,45 & 0,07 \\
\hline Glucosa (mg/dL) & 94,87 & 7,44 & 90,55 & 8,34 & $<0.001$ \\
\hline
\end{tabular}

*Prueba de Rangos de Signos de Wilcoxon

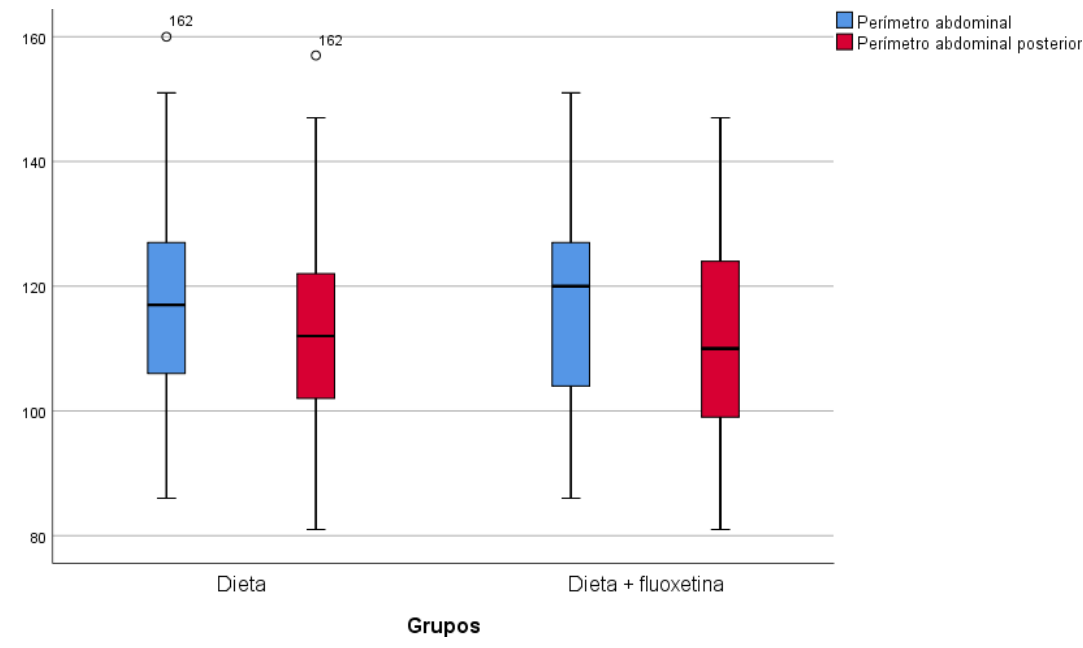

Figura 1. Diagrama de cajas de grupo control y experimental de perímetro abdominal 
Tabla 4

Parámetros antropométricos y bioquímicos antes del inicio del tratamiento y después de seis meses de tratamiento con dieta.

\begin{tabular}{llllll}
\hline & $\begin{array}{l}\text { Antes } \\
\text { Promedio }\end{array}$ & $\begin{array}{l}\text { Después } \\
\text { D.E. }\end{array}$ & $\begin{array}{l}\text { Antes } \\
\text { Promedio }\end{array}$ & $\begin{array}{l}\text { D.E. } \\
\end{array}$ & $\begin{array}{l}\text { Después } \\
\end{array}$ \\
\hline $\begin{array}{l}\text { Peso (kg) } \\
\text { Índice de masa } \\
\text { corporal }\end{array}$ & 40.79 & 7,63 & 39.96 & 7,75 & $<0.001$ \\
$\begin{array}{l}\text { Perímetro } \\
\text { abdominal (cm) }\end{array}$ & 117.63 & 14,59 & 112.63 & 14,66 & $<0.001$ \\
$\begin{array}{l}\text { Insulina (mg/dL) } \\
\text { HOMA-IR }\end{array}$ & 22.64 & 10,20 & 19.82 & 7,66 & $<0.001$ \\
$\begin{array}{l}\text { Triglicéridos } \\
\text { (mg/dL) }\end{array}$ & 5.39 & 2,52 & 4.47 & 1,93 & $<0.001$ \\
$\begin{array}{l}\text { Colesterol } \\
\text { (mg/dL) }\end{array}$ & 158.91 & 68,37 & 137.69 & 31,31 & 0,06 \\
Glucosa (mg/dL) & 95.59 & 6,75 & 90.85 & 7,13 & $<0.001$ \\
\hline
\end{tabular}

*Prueba de Rangos de Signos de Wilcoxon

Tabla 5 .

Parámetros antropométricos y bioquímicos diferenciales y después de seis meses de tratamiento

\begin{tabular}{|c|c|c|c|c|c|}
\hline \multirow{3}{*}{$\begin{array}{l}\text { Valores iniciales - Valores } \\
\text { Finales }\end{array}$} & \multicolumn{4}{|l|}{ Grupos } & \multirow{3}{*}{$\mathrm{p}^{*}$} \\
\hline & \multicolumn{2}{|c|}{ Dieta + fluoxetina } & \multicolumn{2}{|l|}{ Dieta } & \\
\hline & Media & D.E. & Media & D.E. & \\
\hline Diferencial del peso $(\mathrm{kg})$ & 5,85 & 4,50 & 1,21 & 2,26 & 0,03 \\
\hline Diferencial de IMC & 2,54 & 2,02 & 0,83 & 1,33 & 0,00 \\
\hline $\begin{array}{l}\text { Diferencial de Perímetro } \\
\text { Abdominal }(\mathrm{cm})\end{array}$ & 5,16 & 3,91 & 5,00 & 4,49 & 1,00 \\
\hline $\begin{array}{l}\text { Diferencial de Insulina } \\
(\mathrm{mg} / \mathrm{dL})\end{array}$ & $-15,21$ & 7,88 & $-17,32$ & 13,07 & 0,00 \\
\hline Diferencial de HOMA-IR & 0,82 & 1,11 & 0,91 & 3,23 & 0,00 \\
\hline $\begin{array}{l}\text { Diferencial de Triglicéridos } \\
(\mathrm{mg} / \mathrm{dL})\end{array}$ & 35,11 & 96,33 & 21,22 & 76,14 & 1,00 \\
\hline $\begin{array}{l}\text { Diferencial de Colesterol } \\
(\mathrm{mg} / \mathrm{dL})\end{array}$ & 13,60 & 57,43 & 9,11 & 53,38 & 1,00 \\
\hline $\begin{array}{l}\text { Diferencial de Glucosa } \\
(\mathrm{mg} / \mathrm{dL})\end{array}$ & 4,32 & 7,50 & 4,74 & 7,62 & 1,00 \\
\hline
\end{tabular}

*Prueba de Wald-Wolfowitz

\section{CONFLICTOS DE INTERESES}

Los autores no refieren conflictos de intereses 